\title{
Les gisements oubliés du fonds Aragon / Elsa Triolet
}

Entretien mené par Luc Vigier

\section{Olivier Wagner}

\section{OpenEdition}

\section{Journals}

Édition électronique

URL : https://journals.openedition.org/genesis/5257

DOI : $10.4000 /$ genesis.5257

ISSN : 2268-1590

\section{Éditeur :}

Presses universitaires de Paris Sorbonne (PUPS), Société internationale de génétique artistique littéraire et scientifique (SIGALES)

\section{Édition imprimée}

Date de publication : 15 juillet 2020

Pagination : 83-87

ISBN : 979-10-231-0679-4

ISSN : 1167-5101

\section{Référence électronique}

Olivier Wagner, "Les gisements oubliés du fonds Aragon / Elsa Triolet », Genesis [En ligne], 50 | 2020, mis en ligne le 15 juillet 2021, consulté le 03 septembre 2021. URL : http://journals.openedition.org/ genesis/5257 ; DOI : https://doi.org/10.4000/genesis.5257 


\title{
Les gisements oubliés du fonds Aragon / Elsa Triolet Entretien avec Olivier Wagner
}

\author{
Propos recueillis par Luc Vigier
}

[Transcription de l'entretien réalisé le vendredi 26 avril 2019]

Luc Vigier - Pouvez-vous nous dire, pour commencer, où sont localisés les manuscrits d'Aragon et d'Elsa Triolet? Peut-on avoir des certitudes sur les sites qui permettent de consulter ces documents?

Olivier Wagner - Les manuscrits d'Aragon sont assez peu visibles en ligne actuellement. L'inventaire du fonds Aragon / Triolet se trouve sur le Catalogue collectif de France (CCFr), qui n'est pas le plus connu, et en particulier sur la base PALME qui est la base de données des manuscrits et du patrimoine de l'enseignement supérieur et de la recherche. C'est la spécificité de ces archives Aragon / Triolet : elles sont en dépôt à la Bibliothèque nationale de France mais il ne s'agit pas d'un fonds appartenant à cette institution, c'est un dépôt du Centre national de la recherche scientifique. Auparavant, le CNRS disposait de son propre dépôt rue de Richelieu, où le fonds était disposé, un endroit que bien des chercheurs regrettent encore aujourd'hui.

L. V. - Des passerelles numériques ou techniques existentelles entre ce dépôt de la Bibliothèque nationale et par exemple la Bibliothèque Jacques Doucet?

$O$. W. - Non, les archives de la BnF correspondent exactement à ce qui se trouvait chez Aragon quand il a décidé de donner ces archives au CNRS à la fin des années 1970 - pour la déclaration d'intention - et au tout début des années 1980 pour le transfert effectif. Ce qui se trouve chez Doucet, ce sont des pièces dont il s'était séparé de son vivant et en particulier à l'époque de sa collaboration avec Jacques Doucet lui-même. Dans les collections de Doucet, il y a la collection historique, rassemblée par le célèbre mécène lui-même, puis il y a les acquisitions ultérieures, concernant par exemple Aragon, c'est-à-dire des pièces que Doucet avait achetées. Aragon - comme la plupart des surréalistes - ne vivait pas de ses droits d'auteur. Les Dada et les Surréalistes ensuite avaient des diffusions très réduites, ils dépendaient donc pour partie de mécénats et, à l'époque de mécénats avec financement... en échange de manuscrits. Certains se trouvent à la Bibliothèque Jacques Doucet mais Aragon a vendu certains manuscrits à d'autres collectionneurs. Il y a donc des manuscrits d'Aragon qui se trouvent aujourd'hui dans des collections privées.

L. V. - Je pense à la collection Bergé, récemment dispersée. Contenait-elle des manuscrits d'Aragon?

$O$. W. - Bergé était assez peu collectionneur de manuscrits, il était plutôt bibliophile. La seule pièce exceptionnelle provenant de sa bibliothèque était le manuscrit de Nadja, d'André Breton, classé œuvre patrimoniale d'intérêt majeur et acheté par la BnF en 2017. Il était parti en Suisse peu après la publication de l'œuvre en 1928 et Bergé l'avait acheté à Londres en 1998.

L. V. - La nouvelle donne informatique des années 2000 a-t-elle facilité l'accès aux manuscrits et donc aux études génétiques sur l'œuvre d'Aragon?

$O$. W. - La présence en ligne des manuscrits d'Aragon et de Triolet est un sujet dont nous sommes conscients depuis des années, puisque la technologie qui permet la présence en ligne d'une partie de l'inventaire est dépassée depuis longtemps. Ce statut de dépôt CNRS géré par la BnF est un frein, très objectivement, parce que nous n'avions pas les moyens de transférer ces données sur des serveurs équipés de technologies différentes. Notre grand catalogue, pour tout ce qui est hors imprimé, c'est le catalogue Archives et Manuscrits. Il est encodé en langage html, cela permet une interrogeabilité très souple et on peut avoir un aperçu 
de la hiérarchie du classement dans l'arborescence, des ensembles, des sous-ensembles, etc. Or, l'inventaire qui est actuellement dans la base PALME pour le fonds Aragon, c'est un fouillis inextricable. La situation n'est pas bonne mais nous avons maintenant un marché pour rétroconvertir ces bases de données et cela devrait aboutir cette année ou au début de l'année prochaine à la publication en ligne de l'inventaire complet sur cette base Archives et Manuscrits. Cet inventaire inclura évidemment l'inventaire des manuscrits des deux auteurs, mais aussi celui de la correspondance, notamment celle en russe d'Elsa Triolet, qui est très importante...

L. V. - ... dont une bonne partie a été éditée pour la correspondance Elsa Triolet-Lili Brik...

$O$.W. - Oui, mais il y a des ensembles de lettres reçues par Elsa Triolet, qui sont tout aussi importants, notamment des lettres d'amour, des amants qui lui ont écrit - il s'agit-là d'un corpus assez tardif. La correspondance Aragon-Elsa Triolet constitue pour la recherche une mine d'informations, sous-exploitée, et qui ne devrait pas l'être à ce point... Parce qu'il y a des inédits d'un interêt considérable. Je pense en particulier aux lettres d'Henri Matisse à Louis Aragon qui n'ont jamais été éditées ou par exemple, pour Triolet, toutes les lettres d'André Triolet à Elsa (plus d'une centaine).

L. V. - Comment se fait-il? Est-ce parce que le fonds est opacifié par le langage utilisé? Ou bien existe-t-il un interdit sur ces corpus?

$O$. W. - Non, pas d'interdit, c'est plutôt la conséquence d'une tendance lourde de la recherche en sciences humaines aujourd'hui : une exigence de rapidité et d'accès immédiat. À partir du moment où ce n'est pas facilement accessible en ligne à partir d'un moteur de recherche, la consultation patiente des fonds représente une prise de risque très importante, en particulier pour les jeunes chercheurs.

\section{V. - Le temps long de la recherche aurait-il disparu?}

$O . W$. Le recours aux archives est de moins en moins central, en particulier dans le domaine de la littérature. De plus en plus de travaux entiers sur des œuvres, y compris des thèses, se font sans recours aux manuscrits, aux «sources primaires », comme on dirait en Histoire. Certes, c'est un effet de la centralisation parisienne, mais le fonds est aussi victime du flou de ses conditions d'accès. En outre, c'est un fonds qui est soumis à autorisation : il faut passer par moi, je transmets ensuite la demande à Jean Ristat, exécuteur testamentaire, ce qui complique un peu les choses. Le fonds est accessible sur rendez-vous et ce modèle est assez éloigné des pratiques actuelles : écrire à Olivier Wagner, conservateur, archiviste, etc., c'est une procédure plus lourde que la recherche en ligne et cela peut être non seulement contraignant mais aussi déstabilisant.

L. V. - On peut faire un parallèle avec l'IMEC, avec là aussi l'obligation de se déplacer, qui peut intimider. En revanche, une fois qu'on est sur le lieu, tout est accessible.

$O$. W. - L'IMEC impose en plus un isolement, un éloignement... Rue de Richelieu, c'est tout de même plus spontanément accessible. Il faut l'autorisation de Jean Ristat, mais qui est justement très attentif à ce qui se passe autour de ces archives, et je crois qu'il regrette qu'elles ne fassent pas l'objet de plus de travaux.

L. V. - Des continents importants ont-ils échappé aux chercheurs?

$O$. W. - Oui ! C'est d'autant plus étonnant que, contrairement à ce qui s'est passé pour André Breton, avec une sorte d'embargo sur les publications, celui-ci ayant interdit toute édition de sa correspondance pendant cinquante ans, soit de 1966 à 2016, il n'y a jamais eu d'interdit pour la correspondance d'Aragon. La seule raison pour laquelle il n'y a pas plus de travaux d'édition de correspondance, c'est que celle-ci est peu visible. En dehors de cette consultation de la base PALME dans le CCFr, il est impossible de savoir ce qu'il y a dans ces archives. Cette réputation d'inaccessibilité du fonds, exagérée, a pu refroidir des enthousiasmes. Par exemple, les lettres de Vitrac, de Max Morise, de Jean Cocteau : ce sont des dossiers énormes de correspondance.

L. V. - Il n'y a en revanche plus beaucoup d'inédits d'Aragon, pour les æuvres poétiques et romanesques... 
$O$. W. - Les œuvres complètes sont éditées en effet en Pléiade, mais il reste des dossiers en jachères, en particulier sur Les Communistes, Les Incipit et Henri Matisse, roman. Quelques chercheurs s'y sont confrontés ${ }^{1}$, mais il reste beaucoup à découvrir.

L. V. - Dans le fonds Aragon / Triolet, quels sont les dossiers qui vous ont le plus fasciné?

O. W. - Le plus impressionnant, c'est le dossier d'Henri Matisse, roman, justement, un travail énorme de composition, à l'opposition de Traité du style, sans rature ou presque. Pour Henri Matisse, roman, il y a le travail de documentation, les états successifs, à la différence du Paysan de Paris, où l'écriture est plutôt fluide. Pour les romans ultérieurs, c'est beaucoup plus complexe.

\section{V. - Avez-vous fait des acquisitions récemment?}

$O$. W. - Nous sommes très attentifs à ce qui se passe sur le marché. Vous vous souvenez sans doute du scandale Aristophil, qui fut une escroquerie. Il y a donc de nombreux manuscrits qui sont partis dans la nature et en particulier des manuscrits surréalistes. Ces dernières semaines, il y avait une vente importante avec un manuscrit que nous avons cherché à acquérir, une pièce de théâtre de Breton et Aragon, Le Trésor des Jésuites, mais les enchères sont montées trop haut pour que nous puissions en faire l'achat. En revanche, nous avons acquis en 2015 le manuscrit de Traité du style, qui est un très beau manuscrit, même s'il est très peu raturé. Quelques détails permettent de le dater de manière très précise, ce qui atteste que ce n'est pas une copie au propre ou une mise au net. Cela confirme l'extrême facilité d'écriture chez Aragon dont témoigne par ailleurs Breton.

L. V. - A-t-on une idée du prix qu'atteignent les manuscrits d'Aragon?

$O$. W. - Cela peut atteindre quelques dizaines de milliers d'euros. Il y a des collectionneurs et des aragoniens très présents dans le domaine de la collection d'autographes. La liquidation de la SMAF (Société des manuscrits des assureurs français) a généré une arrivée massive de manuscrits surréalistes dans les collections de la BnF, dont celle des Vases communicants de Breton, une version de l'«Entrée des Succubes » (dédié à André Breton et repris dans La Défense de l'infini) et une de «Quelle Âme Divine !» (repris dans Le Libertinage), un roman écrit à six ans... Tout ceci date de 2015 ; il faut ajouter le manuscrit d'Aurélien, acquis dans les années 1980. On a donc d'un côté le fonds et d'un autre les achats effectués par ailleurs, qui font partie des «nouvelles acquisitions françaises». Quatre-vingt-quinze pour cent de ces acquisitions se trouvent dans le dépôt, le reste fait partie de ces «NAF» (nouvelles acquisitions françaises). Un certain nombre de manuscrits appartenaient à Breton et sont passés ensuite par un important collectionneur, René Gaffé. C'est aujourd'hui l'extraordinaire dossier NAF 25097.

\section{V. - De nombreux manuscrits d'Aragon existent également dans le fonds Breton, l'ancien atelier d'André Breton.}

$O . W$. - En effet, au moment de la rupture, Breton a vendu les manuscrits qu'Aragon lui avait donnés. Un énorme dossier est constitué par «L'Affaire Aragon » (NAF 25094) avec le manuscrit de Breton, les brochures, les pamphlets comme «Paillasse!», Misère de la poésie, ainsi que tous les tracts et les formulaires pré-remplis de protestation qui sont signés par des auteurs connus mais aussi par de parfaits inconnus : on repère les réseaux internationaux qui sont à l'œuvre, avec Nancy Cunard, Gide, Cocteau évidemment, mais aussi d'autres personnes moins connues, des textes plus persifleurs ; parfois c'est juste une signature. Il y a un peu de tout, mais c'est une mine d'informations. Autre dossier d'une importance considérable - et tout particulièrement pour la période surréaliste d'Aragon finalement assez peu représentée dans le fonds du CNRS - : l'ensemble des manuscrits offerts par Aragon à Breton (NAF 25097). Au moment de leur rupture, Breton avait vendu cet ensemble à René Gaffé. Transcription en a été faite évidemment dans les Euvres complètes de la Pléiade, mais sans rendre tout à fait justice à la beauté plastique de certaines pages.

1. Voir en particulier Dominique Vaugeois, «Le dossier avant-textue d'Henri Matisse, roman : perspectives sur la genèse d'une composition. Suivi d'un entretien avec Lucie Éon, dactylographe d'Aragon de 1958 à $1972 »$, Genesis, n 21, 2003, p. 95-111 (en ligne sur persee.fr). 
L. V. - Est-ce que la BnF a vocation à initier des travaux de recherche génétique?

$O$. W. - Non, la BnF conserve, elle communique, mais elle n'édite pas directement ses propres collections, même si elle est parfois associée à des projets qui les concernent. Cependant, beaucoup de projets actuels ont trait à la numérisation des manuscrits, dont on sait l'importance pour les chercheurs qui veulent entreprendre des campagnes d'investigation. Un fonds visible en ligne et numérisé et un fonds numérisé invisible en ligne auront des destinées complètement différentes. Aragon, finalement, n'est pas mort il y a longtemps. Son œuvre ne sera libre de droit qu'en 2053. Avant ce moment, toute diffusion en ligne suppose, a priori, une rémunération au titre du droit d'auteur. Le dossier le plus important pour la recherche dans les années à venir me semble vraiment être la correspondance, un gisement de textes extraordinaires. C'est impossible à mettre en ligne évidemment, il faudrait l'autorisation de tous les ayants droit. Mais c'est librement communicable au format papier. Toutes les institutions ne fonctionnent pas de cette manière : à la Bibliothèque littéraire Jacques Doucet, on ne peut consulter la correspondance sans l'autorisation des ayants droit. À la BnF, les collections publiques sont libres d'accès sous condition de présenter un projet de recherche cohérent, dès le niveau master.

L. V. - C'est le souhait qu'avait prononcé Aragon à l'occasion du legs. Il voulait qu'on plonge le regard dans les esquisses, les brouillons mais aussi sans doute dans la correspondance.

$O$. W. - Il y a eu des purges, à l'évidence, certaines choses ont disparu, attestées par Aragon lui-même. De même, une quinzaine de lettres de Breton à Aragon ont disparu à la fin de la vie de ce dernier... Il y a bien sûr ce petit billet de Breton : «Mais Guillaume Apollinaire vient de mourir» et cette autre lettre dans laquelle il dit à Aragon : «Je n'aime que toi.»C'était une amitié masculine, telle qu'on pouvait la concevoir à l'époque, d'une grande intensité. Autre matériau fascinant, les lettres-collages de Breton envoyées à Aragon, d'une beauté à couper le souffle. Datant de 1918 et précédant donc la rédaction des Champs magnétiques, elles semblent pourtant montrer une première exploration de l'automatisme. La correspondance d'Aragon contient de nombreux trésors de ce type.

L. V. - Vous insistez sur la visibilité du manuscrit. Une fois scanné, change pourtant de nature...

$O$. W. - C'est une question centrale des Humanités numériques. Ce passage, ce changement de support, change le rapport au manuscrit, la plupart du temps en bien. Il est plus facile de travailler sur un manuscrit scanné que sur un manuscrit papier, en particulier quand il s'agit d'éditer les textes. La résolution est la plus haute possible. Ce qui est en ligne est un peu plus léger que l'image de base mais la définition est très bonne. Le problème, c'est qu'on peut difficilement mettre en ligne les archives d'auteurs du $\mathrm{xx}^{\mathrm{e}}$ siècle alors qu'il y a une énorme demande de documents numérisés de cette période et en réalité peu de possibilités légales de le faire. Cela a un coût. Des dizaines de milliers d'images sont réalisées, mais cela reste une fraction des collections, peut-être $5 \%$ - problème auquel s'ajoute le coût du copyright. Pour la plupart des documents, ce sera donc sur place que se fera la consultation. Ces numérisations de document sous droits sont cependant consultables à partir du réseau «Gallica intra-muros», dans les locaux de la $\mathrm{BnF}$, depuis Richelieu ou depuis Tolbiac.

Notons pour terminer que parmi les dossiers encore à exploiter, il y a tout le travail d'Elsa Triolet sur Maïakovski. La correspondance traduite Triolet-Brik a laissé l'impression qu'il n'y avait plus rien à étudier. Cependant, même si ce sont des sujets spécifiques, il y a un grand travail à entreprendre sur Elsa Triolet et les écrivains soviétiques. Les cahiers de Triolet sont bien explorés à présent, mais les archives de travail restent un chantier très important, de même que les activités de presse d'Aragon et d'Elsa Triolet.

OLIVIER WAGNER est conservateur à la Bibliothèque nationale de France, au département des Manuscrits. 


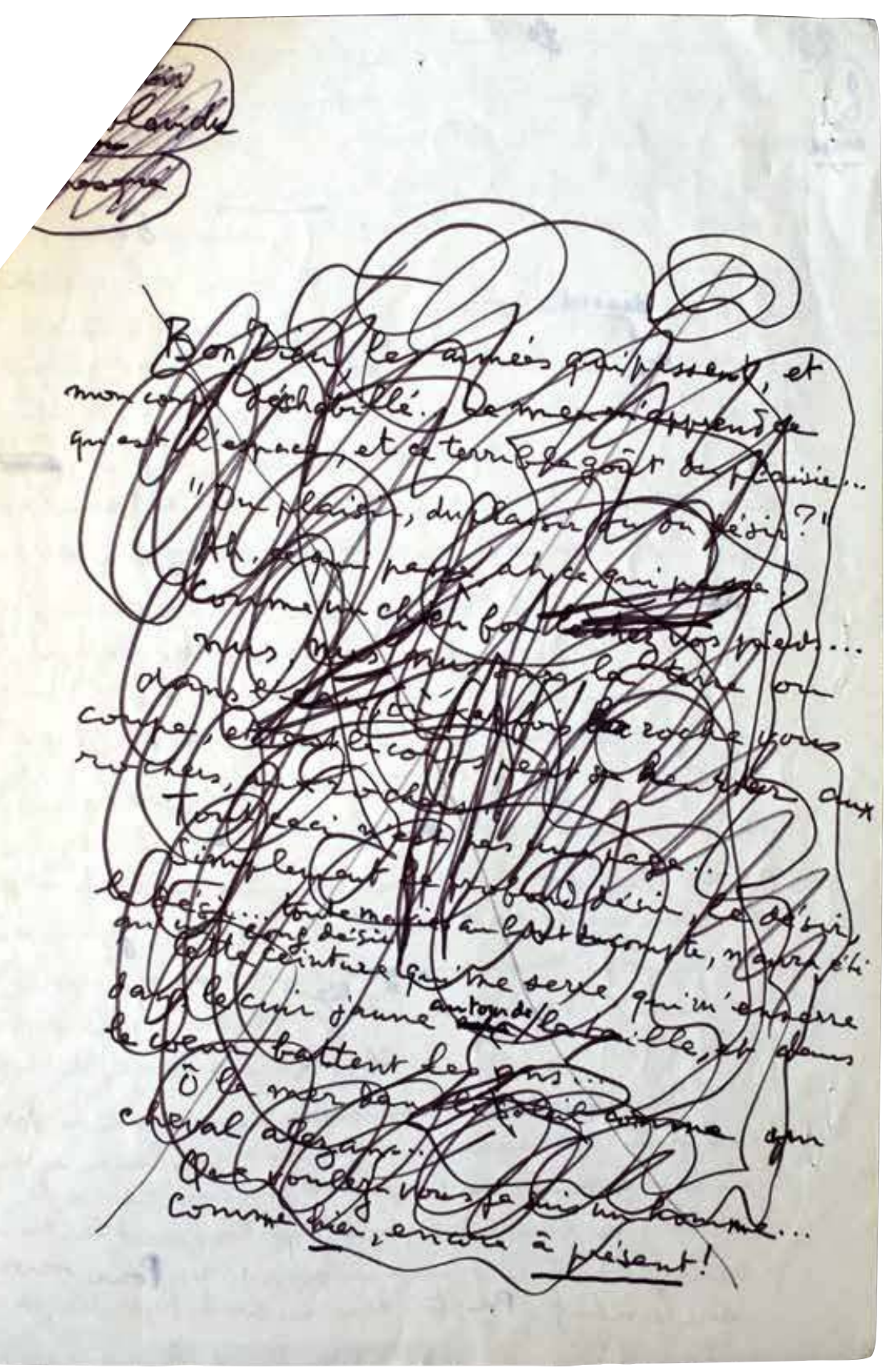

Page manuscrite, raturée et dessinée du discours de 1977.

(๑) Paris, BnF, avec l'aimable autorisation de Jean Ristat 